\title{
Global design practice: IT-based collaboration in AEC-projects
}

\author{
T. Schroepfer \\ Harvard University, USA
}

\begin{abstract}
Information technology is radically and rapidly transforming the way the AEC industry operates. Large, globally operating firms are often out of necessity on the leading edge of implementing advanced IT-tools because their ability to prosper depends more and more on their ability to use technology to augment industry-wide effectiveness and innovation. To maximize the benefits that lie in company-wide highly integrated information and to foster internal collaboration, such companies often rely heavily on the use of IT supporting their practices. As global projects have become the main sources of revenue for many large AEC firms, geographically distributed project teams and human networks have become more and more common. A wide array of tools is available for IT-based collaboration. Global teams and networks face difficulties that are different from those of teams and networks that are not geographically distributed. These difficulties are usually caused by national, cultural, and organizational differences within the organizations and the multitude of markets they are serving as well as the peculiarities of virtual collaboration. This paper examines these phenomena using the Germany-based Hochtief Group, one of the world's largest AEC companies, as an example. It presents a case study of global virtual teamwork in a recently completed large-scale project in Asia. It then moves on to describe Hochtief's participation in a larger research effort initiated by Stanford University's CIFE (Center for Integrated Facility Management) that examines virtual collaboration in the AEC industry. The results of both the case study and the joint research project are analyzed and presented. They suggest a number of important guidelines for global virtual teamwork in the AEC industry, including the suitability of particular IT-tools as well as the coordination of team members with different national, cultural, and organizational backgrounds over multiple time zones.

Keywords: global teamwork, IT-based collaboration, design collaboration, global design practice, global construction practice, AEC project management.
\end{abstract}




\section{Introduction}

More than anything, a division of labor drives processes in the AEC industry. In the sphere where all areas interface, a large cast of characters - clients, architects, contractors, suppliers and many more - work together as a tight-knit ensemble to meet quality expectations, conclude on schedule, and stay within budget limits. This is why coordination and logistics management are so important. Management is foremost a matter of communication, making the use of sophisticated IT an absolute necessity. This becomes all the more vital as more and more companies have a global presence via subsidiaries and associated companies, and accompany their clients into all regions of the world.

Particularly since the early 1970s internationally offered AEC services increased significantly. In 2000, the revenue of the top 225 international AEC companies was approximately USD 116 billion [1]. Companies from the US and Western Europe were the main actors in these markets. Geographically distributed project teams become more and more common in such companies.

\subsection{Hochtief Group}

Hochtief Group is a multinational AEC company with partners and subsidiaries in all continents. Founded in 1875, it is currently Germany's largest building services provider. The organization is one of Europe's leading players in the AEC industry. Following the acquisition of Turner in the USA in 1999 and the engagement in Ambro/BFC in Canada, Hochtief has made the step to one of the world's leading construction companies. In 2001, the company had approximately 40,000 employees worldwide. Its total revenue in 2000 was around USD 12 billion, 76 percent of this revenue was generated with global projects [1].

Together with its subsidiaries and associated companies, Hochtief began gearing for a joint IT/Internet project in late 1999. The keywords of this initiative were "transparency, autonomy, and openness" [2]. The companies were focusing on a number of areas specifying development work that needed to be done. Collaboration and project management of geographically distributed project teams was one of the main areas of the efforts. The goal was to give project participants instant access to the same central data. Since collaboration in the AEC industry relies heavily on time- and cost-intensive communication between a host of partners in far-flung locations - via conventional channels such as phones, fax, paper, and mail. In Hochtief's comparison with conventional tools, Internet-based applications came out as the clear winners: particularly in the design phase, a central electronic project file could facilitate the exchange of data. Up-to-the minute information could be available to all parties at all times. Geographical distances would no longer be important because everyone involved could use a standard web browser to access the same project file wherever and whenever necessary. The system's workflow capabilities would ensure documentation of individual process steps and continuous updates on status 
information would allow monitoring and keep projects on track. The 1999 initiative focused on the technical aspects of global collaboration. However, practice showed that the issues of global collaboration were more complex.

\subsection{The Taiwan High Speed Rail project}

The Taiwan High Speed Rail Project was a typical global AEC project for HOCHTIEF. A high-speed train rail connection of approximately 40 kilometers in length, the project included the building of several bridges as well as a train depot. The project was located in an area with a high earthquake risk. The project team included a British office, several offices in Taiwan and India, a contractor's independent checking engineer in Denmark and numerous other experts and consultants located all over the world [3].

\subsubsection{Steering of design and construction}

In a project like Taiwan High Speed Rail, the early recognition of problems was crucial. This meant a continuous

- $\quad$ questioning of executed project parts

- discussion of typical problems

- discussion of technical standards

- discussion of local practice

- $\quad$ questioning of appropriateness of software tools

- Exchange of drawing samples to clarify graphic standards

\subsubsection{Design quality}

Quality requirements had to be checked systematically:

- design quality plan: illustration of the project organization with responsibilities

- design manual: communication with client and subcontractors

- CAD manual: requirements of client

- quality checks: always in house at HOCHTIEF in Germany

- construction requirements: examples for standard details for subcontractors

\subsubsection{Document management}

Approximately 12,000 drawings were produced over the course of the project. Each drawing had to be revised about 2-3 times which lead to a total number of approximately 30,000 drawings that were sent electronically to project partners and subcontractors in Taiwan, Germany, England, India, Malaysia, and Denmark. All other documents - calculations, meeting minutes, contracts, correspondence, etc. - were handled in the same manner. Project servers were located in Germany, Taiwan, and Denmark. The servers were synchronized several times during each day so that all project participants had the same information status at all times.

In summary, the Taiwan High Speed Rail Project showed many characteristics of global AEC projects: 
- project teams geographically distributed

- different time zones

- different cultures and languages

- IT-based communication

- different national standards

The wish to better understand the peculiarities of such a highly IT-based collaboration led HOCHTIEF to the participation of Stanford University's Global Teamwork Project in 2001.

\section{Global Teamwork project}

The master builder's atelier in the information age was the vision behind an integrated research and curriculum in AEC Global Teamwork offered by the Department of Civil and Environmental Engineering at Stanford University in 2001 [4]. The project examined the use of modern communication technologies to promote better collaboration in projects where team members are geographically separated from one another. The objectives of Global Teamwork project were to develop, test, deploy, and assess new Workspaces and IT processes, including learning, work culture, and new approaches to multidisciplinary collaborative, geographically distributed teamwork

The goal was also to educate students as the next generation of design and building professionals to know how to team up with professional from other disciplines using IT and to leverage collaboration and IT to improve processes as well as products.

The core of the project was the "AEC Team": students who played the role of the apprentice, AEC graduate students and who played the role of the journeyman, faculty members and researchers who played the role of the "master builders", and industry members who played the role of mentors, owners, and sponsors. The project engaged 43 students from 10 universities worldwide (20 from Stanford and 23 from other university partners) in twelve AEC teams USA: Stanford, UC Berkeley, Kansas University, Georgia Tech; Europe: TU Delft (The Netherlands), ETH Zurich and FH Aargau (Switzerland), Bauhaus University (Germany), Ljubljana University (Slovenia) and Stanford Japan Center. The participants were challenged to cross four chasms:

- AEC cross-disciplinary project-based teamwork

- use of information and collaboration technology

- team coordination over multiple time zones

- team coordination over multiple cultures

Each team was geographically distributed over two or three time zones. The Stanford lab computational infrastructure offered the necessary spectrum of information and collaboration technologies, such as video-conferencing, videostreaming, web-based collaboration applications team discussion forum, 4D$\mathrm{CAD}$, project group spaces, Internet 2 , wireless and mobile infrastructure.

The project started in January 2001 with a kick-off event that brought together all the students, faculty, owners, and mentors at Stanford University. 
During that time students engaged in team building exercises, met their "owners" and were introduced to the project and basic collaboration technologies. After the kick-off all students went back to their campuses and for five months learned and worked in cyberspace. A halfway milestone was the concept development presentation event that took place in cyberspace via video-conferencing and application sharing. Students, faculty and mentors experienced presentations and discussions of projects in a global environment. Some industry members connected to this event from their trailers on a construction site. The project culminated in a final team project presentation in May when all students, faculty, owners, and mentors came to Stanford again to share their products, processes, experiences, lessons learned. The Global Teamwork project showed remarkable parallels to the demands placed on construction teams in the framework of largescale global projects in the AEC industry as described in the example of the Taiwan High Speed Rail project.

\subsection{Experiences with the Global Teamwork project}

After the end of the Global Teamwork project, Hochtief invited all participants to a workshop at its headquarters in Essen, Germany, to discuss the experiences gathered in the project and to identify improvement potentials for collaboration in global project teams. The following is a summary of the main issues that were raised by the participants:

\subsubsection{Time for technical issues}

A wide range of tools were available for the communication in meetings and events of the Global Teamwork Project. Especially audio and NetMeeting turned out to be problematic. Dealing with technical problems required $50-60 \%$ of the team meeting time in the beginning of the project. This percentage was lowered to $10-20 \%$ as the project proceeded. The real problem turned out to be the poor audio connections that significantly affected the quality of the communication in the meetings.

\subsubsection{Time for communications tools}

The geographically distributed teams relied on IT communication. Clarification of project issues using NetMeeting required approximately $30-40 \%$ more time as if the team members would have sat together physically.

\subsubsection{Getting familiar with software}

Many project participants had to learn new software. Requirements were the creation of a homepage, 3D and 4D models, and PowerPoint presentations. The communication tools, FTP, AutoCAD, MS Office, etc. were required as a standard. Depending on the individual experience of the participants it took up to several weeks until everyone handled the software efficiently.

\subsubsection{Suitability of IT tools}

Since the project had about 40 participants, technical requirements had to be clarified in advance. The following tools were the standard to guarantee collaboration: 
- $\quad$ MS Office 2000

- Frontpage 2000

- AutoCAD 2000, including ADT (Architectural Desktop)

- 3D Studio Max

- Adobe Photoshop

- Video conference system

- FTP

- Internet access

- ISDN for audio conferences

- Specific versions of Internet Explorer

- Specific versions of NetMeeting

- Software of the several project disciplines (cost estimation, etc.)

- Common data storage

\subsubsection{Teams}

Personnel requirements turned out to be at as least as important as technical requirements. Crucial were:

- reliability

- communication skills

- $\quad$ fluent project language (English)

- impartiality

- openness

- pro-activeness

- motivation and commitment

It turned out to be beneficial to allow the establishment of personal contacts before the start of the actual project. It paid off to have common relaxing activities as well as playful tests of collaboration. It became clear that it took time to bridge differences of language, culture and personalities. It also became clear that the better the initial phase of personal meetings was, the better the actual project collaboration was.

Team members had to be clear from the very beginning that they had to share all relevant data with their colleagues. It was important that the members explained each other what their work, their goals, their thoughts, and their proposed solutions were. Interaction only took place when members understood the concerns and ways of thinking of the other disciplines. The skills of forming a good team required the willingness to interact with each other already in the conceptual phase of the project.

One of the most important issues of the project was the organization of teamwork. It was important for the teams had to have a project manager. Project documentation had to be detailed and always up to date. A project homepage proved to be a good tool.

Bringing together the various disciplines already during the conceptual design phase turned out to be essential. The requirements of the various disciplines then had an impact on the project from the very beginning. This improved the quality of the team. Each team member stood behind the project with discipline and 
motivation. Generating requirement lists of the single disciplines proved especially helpful. These were lists of things one would like, common to-do lists, etc.

Information exchange had to be carefully organized. On one hand this was a technological problem: how and in what time intervals the information exchange occurred, how and in what software version information was stored etc. On the other hand this was a content problem: what kind of information was necessary at what time and what kind of decision had to be made by the whole team and what not. What had to be handled in a synchronous manner and what could be handled in an asynchronous manner?

It turned out to be important to develop a common structure for all participants in the beginning of the project. Competences and responsibilities had to be clarified but still be kept flexible to a certain degree. Team processes like communication, flow of information, design, etc. had to be carefully organized and structured. Different time zones turned out to be difficult for scheduling meetings for all participants.

\section{Conclusions}

During and after the workshop at Hochtief's headquarters in Essen, Germany, the Global Teamwork participants discussed which were the most important lessons learned for global IT-based collaboration. The following is a summary of what the participants felt were the most crucial issues.

\subsection{Communication}

Communication happened on the level of relevant information as well as on the level of emotional relationships between the team members. Any kind of information exchange caused emotional reactions and influenced the quality of collaboration. The project showed that the spatial distribution of the team members reinforced these effects. The project participants agreed that it is an essential advantage for a geographically distributed team to have an initial phase during that it was possible to physically meet. The geographical distribution of the members had to be compensated with more intense communication using the many different IT tools.

\subsection{Trust}

Because of the geographical distribution and the resulting anonymity within the teams the development of trust through openness, helpfulness, and tolerance seemed even more essential for the success of the projects.

\subsection{Structure of teams}

Having a team leader and inventing rules for collaboration turned out to be even more important as if the team would have worked together in the same location. 


\subsection{Structure of tasks}

The same was felt for the structure of tasks: clearly and early articulated time frames, goals, milestones, distinction of responsibilities, and level of processing turned out to be especially helpful.

\subsection{Excellent technical equipment}

The elixir of life in the project was intense communication. If this was not guaranteed, frustration and disintegration occurred within the teams. IT therefore had to be excellent in the sense of being reliable.

\subsection{Goal-oriented information management}

The answer to the following question turned out to be essential for the success of the projects: which kind of information should be transmitted when and in what depth through whom to whom? It was an ideal situation when each member of the team had the same information status at all times.

\section{References}

[1] Engineering News Record, The Top 225 International Contractors, McGraw Hill: New York, pp. 66-87, 2001

[2] Hochtief Group, Baubude Express July 2000, Hochtief Communications: Essen

[3] In February 1999, Hochtief also founded a technical office in India that offers services for all divisions of the company. Since then, the staff has grown significantly. Services range from the creation of reinforcement drawings all the way to quantity surveys for major construction projects. They also provide support in material procurement. Depending on the location of the project, the team in India provides German, British, US, or Australian standards.

[4] The project, headed by Prof. Renate Fruchter, was part of a continuous research program of Stanford University's Department of Civil and Environmental Engineering that was established in 1993. 